Annals of Pure and Applied Mathematics

Vol. 14, No. 1, 2017, 39-44

ISSN: 2279-087X (P), 2279-0888(online)

Published on 25 June 2017

Annals of

www.researchmathsci.org

DOI: http://dx.doi.org/10.22457/apam.v14n1a5

Pure and Applied

Mathematics

\title{
Line Set Dominating Set with Reference to Degree
}

\section{P.Solai Rani ${ }^{1}$ and R.Poovazhaki ${ }^{2}$}

${ }^{1}$ Department of Mathematics, R.V.S.College of Arts and Science, Sulur Coimbatore Tamil Nadu, India

Corresponding author. Email: prsolairani@gmail.com

${ }^{2}$ Principal, E.M.G.Yadava Women's College, Madurai, Tamil Nadu, India

Email: rpoovazhaki@yahoo.co.in

Received 12 May 2017; accepted 2 June 2017

Abstract. A set $D^{l} \subseteq E(G)$ is said to be a Strong Line Set Dominating set (slsd-set) of G. If for every set $R \subseteq E-D^{l}$. There exists an edge $e \in D^{l}$, such that the sub graph $\langle R \bigcup\{e\}\rangle$ is induced by $R \bigcup\{e\}$ is connected and $d(e) \geq d(f)$ for all $f \in R$ where $d(e)$ denote the degree of the edge. The minimum cardinality of a $s l s d$-set is called the strong line set dominating number of $\mathrm{G}$ and is denote by $\vartheta_{s l}^{\prime}(G)$. In this paper Strong Line set Dominating set are analyse with respect to the strong domination parameter for separable graphs. The characterization of separable graphs with $s l s d$ number is derived.

Keywords: Separable graph, line set dominating set, strong line set dominating set.

AMS Mathematics Subject Classification (2010): 05C69

\section{Introduction}

Domination is an active subject in graph theory. Let $G=(V, E)$ be a graph. A set $D \subseteq V(G)$ of vertices in a graph $G=(V, E))$ is a dominating set. if every vertex in $V-D$ is adjacent to some vertex in D. The domination number $\gamma(G)$ of $\mathrm{G}$ is the minimum cardinality of dominating set in $\mathrm{G}$. A dominating set $\mathrm{D}$ is called a minimal dominating set if no proper subset of $\mathrm{D}$ is a dominating set $[3,4]$.

Let $G=(V, E)$ be a graph. A set $F \subseteq E(G)$ is an edge dominating set of $\mathrm{G}$. if and only if every edge in E-F is adjacent to some edge in $\mathrm{F}$. The edge domination number $\gamma^{\prime}(G)$ is the minimum of cardinalities of all edge dominating sets of G.[4]

A dominating set $\mathrm{S}$ is a strong dominating set if for every vertex $\mathrm{u}$ in $\mathrm{V}-\mathrm{S}$, There is a vertex $\mathrm{v}$ in $\mathrm{S}$ with $\operatorname{deg}(\mathrm{v}) \geq \operatorname{deg}(\mathrm{u})$ and $\mathrm{u}$ is adjacent to $\mathrm{v}[1,2]$.

Let $\mathrm{G}$ be a graph. A set $D \subseteq V(G)$ is a point set dominating set (PSD-set) of G. if for each set $S \subseteq V-D$, there exists a vertex $u \in D$ such that the sub graph $\langle S \cup\{u\}\rangle$ induced by $S \cup\{u\}$ is connected. The point set domination number (PSD-number) $\gamma_{p}^{\prime}(G)$ of $\mathrm{G}$ is the minimum cardinalities of all PSD-Set of G.[6] 


\section{P.Solai Rani and R.Poovazhaki}

Let $\mathrm{G}$ be a graph. A set $D \subseteq V(G)$ is said to be a strong point set dominating set (spsd-set) of G. if for each set $S \subseteq V-D$, there exists a vertex $u \in D$ such that the sub graph $\langle S \cup\{u\}\rangle$ induced by $S \cup\{u\}$ is connected and d(u) $\geq d($ s) for all s $\in S$ where $\mathrm{d}(\mathrm{u})$ denote the degree of the vertex u. The Strong point set domination number (spsd) $\gamma_{s p}^{\prime}(G)$ of $\mathrm{G}$ is the minimum cardinalities of all spsd-set [7, 8].

Rao and Vijayalakmi introduced the concept of Line set domination set and derived results parallel to those of Sampathkumar and Pushpalatha [5].

Let $\mathrm{G}$ be a graph. A set $F \subseteq E(G)$ is a line set dominating set (lsd-set) of G, if for each set $S \subseteq E-F$, there exists an edge $e \in F$ such that the sub graph $\langle S \cup\{e\}\rangle$ is induced by $S \cup\{e\}$ is connected. The line set domination number $v_{l}^{\prime}(G)$ (lsd-number) is the minimum cardinalities of all lsd-set of $\mathrm{G}$.

Let $x, y$ in $E(G)$ of an isolates free graph $\mathrm{G}(\mathrm{V}, \mathrm{E})$, then an edge $\mathrm{x}$, e-dominates an edge if $\mathrm{y}$ in $\langle N(x)\rangle$. A line graph $L(G)$ is the graph whose vertices corresponds to the edges of $G$ and two vertices in $L(G)$ are adjacent iff the corresponding edges in $G$ are adjacent $(\mathrm{V}(\mathrm{L}(\mathrm{G}))=\mathrm{q})$. For any edge e, let

$$
N^{\prime}(e)=\{e \in F: e \text { and } f \text { have a vertex in common }\}
$$

and $N^{\prime}[e]=N^{\prime}(e) \bigcup\{x\}$. For a set $F \subseteq E(G)$ Let $N^{\prime}(F)=\bigcup N^{\prime}(e)$. The degree of an edge $\mathrm{e}=\mathrm{uv}$ of $\mathrm{G}$ is defined by $\operatorname{deg}(\mathrm{e})=\operatorname{deg}(\mathrm{u})+\operatorname{deg}(\mathrm{v})-2$. The maximum and minimum degree among the edge of graph $\mathrm{G}$ is denote by $\Delta^{\prime}(G)$ and $\lambda(G)$ (the degree of an edge is the number of edges adjacent to it) A connected graph with at least one cut edge is called a separable graph. That is an edge e such that $\mathrm{G}-\mathrm{e}=\{\mathrm{E}-\{\mathrm{e}\}\}$ is disconnected [4].

\section{Results and bound}

Definition 2.1. A set $D^{l} \subseteq E(G)$ is said to be a strong line set dominating set (slsd-set) of G. If for every set $R \subseteq E-D^{l}$. There exists an edge $e \in D^{l}$, such that the sub graph $\langle R \bigcup\{e\}\rangle$ is induced by $R \bigcup\{e\}$ is connected and $d(e) \geq d(f)$ for all $f \in R$ where $d(e)$ denote the degree of the edge. The minimum cardinality of a slsd-set is called the Strong Line Set Dominating Number of $\mathrm{G}$ and is denote by $\vartheta_{s l}^{\prime}(G)$.

Theorem 2.2. If a connected graph $\mathrm{G}$ with $\mathrm{n}$ edge, then

$$
\vartheta^{\prime}(G) \leq \vartheta_{l}^{\prime}(G) \leq \vartheta_{s l}^{\prime}(G) \leq q-\Delta^{\prime}(G) \text { where } \Delta^{\prime}(G) \text { is the maximum degree of } \mathrm{G} \text {. }
$$

Proof: Since every slsd-set of $\mathrm{G}$ is line set dominating set and we known that every line set dominating set of $\mathrm{G}$ is a edge dominating set of $\mathrm{G}$ and Let e be a edge of maximum degree $\Delta^{\prime}(G)$. Then $\mathrm{e}$ is adjacent to $N^{\prime}(e)$, such that $\Delta^{\prime}(G)=N^{\prime}(e)$. Hence $E-N^{\prime}(e)$ is a slsd- set. Therefore $\vartheta_{s l}^{\prime}(G) \leq\left|E-N^{\prime}(e)\right|$. Hence $\vartheta^{\prime}(G) \leq \vartheta_{l}^{\prime}(G) \leq \vartheta_{s l}^{\prime}(G) \leq q-\Delta^{\prime}(G)$.

In this next result, we list the exact values of $\vartheta_{s l}^{\prime}(G)$ for some standard graphs. 
Line Set Dominating Set with Reference to Degree

Observation 2.3. For any complete graph $K_{n}$, then

$$
\vartheta_{l s}^{\prime}(G)=\left\{\begin{array}{ll}
\frac{n}{2} & \text { for any positive even int eger } \\
\frac{n-1}{2} & \text { for any positive odd int eger }
\end{array}\right\}
$$

Observation 2.4. For any star $K_{1}, n-1$, then

$$
\vartheta_{s l}^{\prime}\left(K_{1, n-1}\right)=1 \text {. }
$$

Observation 2.5. For any path $\mathrm{P}_{n}$, then

$$
\vartheta_{s l}^{\prime}\left(P_{n}\right)=\left\{\begin{array}{ll}
1 & n=3,4 \\
3 & n=5 \\
n-3 & n \geq 6
\end{array}\right\}
$$

Observation 2.6. For any cycle $\mathrm{C}_{\mathrm{n}}$, then

$$
\vartheta_{s l}^{\prime}\left(C_{n}\right)=\left\{\begin{array}{ll}
1 & n=3 \\
2 & n=4,5 \\
n-2 & \text { for any positive int eger } n \geq 6
\end{array}\right\} .
$$

Observation 2.7. If $\left(K_{n, m}\right)$ is a complete bi-partite graph of $\mathrm{m}, \mathrm{n}>2$ vertices, then

$$
\vartheta_{s l}^{\prime}\left(K_{n, m}\right)=\left\{\begin{array}{ll}
\frac{m+n}{2} & \text { for } m=n \\
\frac{m+n-1}{2} & \text { for } m<n
\end{array}\right\} .
$$

In the next result, Strong line set dominating set in graph $G$ is the Strong Point set dominating number of the line graph $L(G)$.

Observation 2.8. For any path $\mathrm{P}_{w}$, for any positive integer $\mathrm{n} \geq 5$ vertices $\vartheta_{s l}^{\prime}\left(P_{n}\right)=\gamma_{s l}\left(L\left(P_{n}\right)=\gamma_{s l}\left(P_{n-1}\right)=n-3\right.$.

Observation 2.9. For any cycle $C_{n}$, for any positive integer $n \geq 5$ vertices

$$
\vartheta_{s l}^{\prime}\left(C_{n}\right)=\gamma_{s p}\left(L\left(C_{n}\right)=\gamma_{s p}\left(C_{n}\right) .\right.
$$

Observation 2.10. For any star $K_{1, n}$ for any positive integer with $\mathrm{n} \geq 2$ vertices

$$
\vartheta_{s l}^{\prime}\left(L\left(K_{1, n}\right)\right)=\vartheta_{s l}^{\prime}\left(K_{n-1}\right) \text {. }
$$


P.Solai Rani and R.Poovazhaki

Theorem 2.11. Let $\mathrm{G}$ be a connected graph and $D^{l} \subseteq E(G)$ be a strong line set dominating set of G. then for every subset $R \subseteq E-D^{l}$ in $\bigcup_{e \in D^{l}}\left\langle N^{\prime}(e)\right\rangle$. There exists an edge $e \in D^{l}$, such that $\langle E-N(r)\rangle$ for all $r \in R$ is maximal edge dominating set.

Lemma 2.12. Let $G(V, E)$ be any graph and $D^{l}$ be any strong line set dominating set. Then $\left(E-D^{l}\right)$ is a proper sub graph of a component $H(G)$.

Proof: Suppose there exists e and $f$ belonging to two different components of G. Since $D^{l}$ is a strong line set dominating set of G. There must exists $w \in D^{l}$, such that $\langle e, f, w\rangle$ is connected and $d(w) \geq d(f)$ for all $f \in E-D^{l}$. Contrary to the assumption, This implies $E-D^{l} \subseteq E(H)$ for some component $\mathrm{H}$ of $\mathrm{G}$. Further, since $D^{l}$ is a sls-dominating set of $D^{l} \cap E(H) \in \vartheta_{s l}^{\prime}(H)$. Hence $D^{l} \cap E(H)=\phi$, which implies that $(E-F)$ is a proper sub graph of $\mathrm{H}$.

Theorem 2.13. Let $\mathrm{G}$ be a finite graph of order $\mathrm{n}$, and $C_{G}$ denote the set of its components. Then

$$
\vartheta_{s l}^{\prime}(G)=q-\max _{H \in C_{G}}\left\{E(H)-\vartheta_{s l}^{\prime}(H)\right\} .
$$

Proof: Let $D^{l}$ be a $\vartheta_{s l}^{\prime}(G)$ G. By lemma 2.12 it follows that there exists $H \in C_{G}$. such that $E-D^{l} \subseteq E(H)$. Clearly $D^{l} \cap E(H) \in \vartheta_{s l}^{\prime}(H)$ and since

$$
\left|D^{l}\right|=\left|D^{l} \cap E(H)\right|+q-|E(H)|
$$

We have $\vartheta_{s l}^{\prime}(G) \geq q-|E(H)|+\vartheta_{s l}^{\prime}(H) \Rightarrow \vartheta_{s l}^{\prime}(G) \geq q-\max \left\{E(H)-\vartheta_{s l}^{\prime}(H)\right\}$

On the other hand,

$$
\vartheta_{s l}^{\prime}(G) \leq q-\left|E(G)-\vartheta_{s l}^{\prime}(H)\right| \Rightarrow \vartheta_{s l}^{\prime}(G) \geq q-\max \left\{E(H)-\vartheta_{s l}^{\prime}(H)\right\}
$$

From inequalities [3] and [4], we have $\vartheta_{s l}^{\prime}(G)=q-\max _{H \in C_{G}}\left\{E(H)-\vartheta_{s l}^{\prime}(H)\right\}$

In the remaining discussion of this paper, a graph $\mathrm{G}$ always means a separable graph.

Observation 2.15. If $\mathrm{G}$ is separable graph with sls-dominating set $\mathrm{S}$. Then $B \cap D^{l}$ is a sls-dominating set of $\mathrm{B}$ for any block $B \in B_{G}$. (where $B_{G}$ is the set of all blocks.)

Proof: Let $T \subseteq B-B \cap D^{l}$. Then that $T \subseteq E-D^{l}$ and $D^{l}$ is a $s l s d$-set. Therefore there exists $e \in D^{l}$ such that $T \subseteq N(e)$. Hence e is adjacent to more than one edge in B and $d(e) \geq d(t) \quad \forall t \in T(G)$. i.e. $e \in B \cap D^{l}$. Therefore $B \cap D^{l}$ is a slsd-set of B.

Observation 2.16. If a block $B$ has a slsd-set $B$ containing all cut edge belonging to $B$ Then $(E-B) \cup B^{\prime}$ is a slsd- set . 


\section{Line Set Dominating Set with Reference to Degree}

Remark 2.17. If $D^{l}$ is an $\vartheta_{s l}^{\prime}(G)$ set of separable graph, then there are two cases:

i) $\mathscr{D}_{s l}(\mathrm{G}: \mathrm{X})=\left\{D^{l} \in D_{s l}(G): \exists a B \in B_{G}\right.$ with $\left.E-D^{l} \subseteq E(B)\right\}$

ii) $\mathscr{D}_{s l}(\mathrm{G}: \mathrm{Z})=\left\{D^{l} \in D_{s l}(G): E-D^{l}\right.$ contain edges of different blocks $\}$

Definition 2.18. Let $\mathrm{G}=(\mathrm{V}, \mathrm{E})$ be any graph with cut vertices, $D^{l} \in \mathcal{D}_{s l}(\mathrm{G})$ and $E-D^{l} \subseteq E(B)$,

Define $L\left(B, D^{l}\right)=\left\{e \in E-D^{l}: N(e) \cap\left(D^{l} \cap E(B)\right\}\right.$.

Remark 2.19. If $L\left(B, D^{l}\right) \neq \phi$ then $E(B) \cap D^{l} \in \mathcal{D}_{s l}$ (B). This yields, $\left|B \cap D^{l}\right|$ $<\vartheta_{s l}^{\prime}(B)$. This, in fact, we have

$$
L\left(B, D^{l}\right) \neq \phi \Rightarrow \vartheta_{s l}^{\prime}(G)=n-\Delta^{\prime}(G)
$$

Theorem 2.20. If $L\left(B, D^{l}\right) \neq \phi$, then $\vartheta_{s l}^{\prime}(G)=q-k_{s l}$. where $k_{s l}=\max \left\{E(B)-\vartheta_{s l}^{\prime}(B)\right\}$.

Proof: Let $L\left(B, D^{l}\right) \neq \phi \quad$ implies $B \cap D^{l}$ is a slsd set of $\mathrm{B}$ and hence $\vartheta_{s l}^{\prime}(B) \leq\left|B \cap D^{l}\right|$ Also $\vartheta_{s l}^{\prime}(B) \geq\left|B \cap D^{l}\right|$. For, if $\vartheta_{s l}^{\prime}(B) \leq\left|B \cap D^{l}\right|$, then $(E-B) \cup B^{\prime}$ is a slsd set of $\mathrm{G}$ where $\left|B^{\prime}\right|=\vartheta_{s l}^{\prime}(B)$. Then $\left|D^{l}\right|=(E-B) \cup\left(B \cap D^{l}\right) \geq\left|(E-B) \cup B^{\prime}\right|$. That is, there exists a slsd set $(E-B) \cup B^{\prime}$ of $\mathrm{G}$ with cardinality less than equal to $\left|D^{l}\right|$ which is a contradiction. Hence

$$
\begin{aligned}
& \vartheta_{s l}^{\prime}(B) \geq\left|B \cap D^{l}\right| . \text { Therefore, } \vartheta_{s l}^{\prime}(B)=\left|B \cap D^{l}\right| . \text { Hence } \\
& \vartheta_{s l}^{\prime}(G)=\left|D^{l}\right|=\left|(E-B) \bigcup\left(B \cap D^{l}\right)\right|=\left|(E-B) \bigcup B^{\prime}\right| \geq q-k_{s l} .
\end{aligned}
$$

Therefore, $\vartheta_{s l}^{\prime}(G)=q-k_{s l}$.

\section{Remark 2.21.}

i) $\mathcal{D}_{s l}\left(\mathrm{G}: \mathrm{X}_{1}\right)$ denotes the set of all slsd-set $\mathrm{F}$ of $\mathrm{G}$ with $E-D^{l} \subseteq E(B)$ and $L\left(B, D^{l}\right) \neq \phi$ for some $\mathrm{B} \in \mathscr{B}_{\mathrm{G}}$.

ii) $\mathcal{D}_{s}\left(\mathrm{G}: \mathrm{X}_{1}\right)$ denotes the set of all slsd-set $\mathrm{F}$ of $\mathrm{G}$ with $(E-F)-L(B, F) \neq \phi$.

Theorem 2.21. $\mathscr{D}_{s l}\left(\mathrm{G} ; \mathrm{X}_{1}\right) \neq \varnothing$ if and only if $\Delta^{\prime}(G) \leq k_{s l}+1$.

Proof: Let $\left.D^{l} \in \mathcal{D}_{s}\left(\mathrm{G} ; \mathrm{X}_{1}\right) \neq \varnothing\right)$, Then by definition of $\mathcal{D}_{s}\left(\mathrm{G}: \mathrm{X}_{1}\right)$ there exist $B \in B_{G}$ such that $E-D^{l} \subseteq E(B)$ for some block $\mathrm{B}$ of $\mathrm{G}$ and $L\left(B, D^{l}\right) \neq \phi$, Also, 
P.Solai Rani and R.Poovazhaki

$E(B) \cap D^{l}$ is slsd-set of $B-L\left(B, D^{l}\right)$. By the definition of $L\left(B, D^{l}\right)$, one can easily see that $\left(E(B) \cap D^{l}\right) \bigcup\{e\}, e \in L\left(B, D^{l}\right)$ is a slsd-set for B so that

$$
\vartheta_{s l}^{\prime}(B) \leq\left|B \cap D^{l}\right|+1
$$

Also, $\quad\left|B \cap D^{l}\right| \leq \vartheta_{s l}^{\prime}(B)$

For otherwise, $\{E(G)-E(B)\} \bigcup \vartheta_{s l}^{\prime}(G)$ would be a slsd-set of $\mathrm{G}$ having less than $\left|D^{l}\right|$ edges contrary to the fact $D^{l}$ is a $\vartheta_{s l}^{\prime}(G)$ By (1) and (2). We get

$$
\vartheta_{s l}^{\prime}(B)-1 \leq\left|B \cap D^{l}\right| \leq \vartheta_{s l}^{\prime}(B)
$$

Now, $\left|D^{l}\right|=n-|E(B)|+\left|B \cap D^{l}\right| \geq n-|E(B)|+\vartheta_{s l}^{\prime}(B)-1$. That is

$$
n-\Delta^{\prime}(G) \geq n-|E(B)|+\vartheta_{s l}^{\prime}(B)-1
$$

Or, equivalently, $\Delta^{\prime}(G) \leq|E(B)|-\vartheta_{s l}^{\prime}(B)+1 \leq k_{s l}+1$. Thus, we have $\mathcal{D}_{s l}\left(\mathrm{G} ; \mathrm{X}_{1}\right) \neq \varnothing$, $\Rightarrow \Delta^{\prime} \in\left\{k_{s l}, k_{s l}+1\right\}$.

Acknowledgement. The authors are graceful to the referees and editors whose valuable suggestions resulted in a better organized and improved paper.

\section{REFERENCES}

1. B.D.Acharya and P.Gupta, On point-set domination in graphs, in; Proc. Nat. Seminar on Recent Development in Mathematics, N.M. Bujurke (Ed.), Karnataka University Press, Dharwad, 1996,106-108.

2. B.D.Acharya and P.Gupta, On point set domination in graphs: IV Separable graphs with unique minimum psd-sets, Discrete Mathematics, 195 (1999) 1-13.

3. F.Harary, Graph theory, Addison - Wesley, 1969.

4. T.Haynes, S.T.Hedetniemi and P.J.Slater, Fundamentals of domination in graphs, Marcel Dekker Inc, New York, 1998.

5. I.H.Nagaraja Rao and B.VijayaLaxami, Line set domination number of a graph, Indian J. Pure Appl. Math., 30(7) (1999) 709-713.

6. E. Sampathkumar and L. Pushpalatha, Point set domination number of a graph, Indian J. Pure Appl. Math., 24(4) (1993) 225 - 229.

7. E.Sampathkumar, Graph Theory Research Report No:3, Karnatak University, Dharwad, 1972.

8. E.Sampathkumar and L.Pushpalatha, Strong weak domination and domination balance in a graph, Discrete Math.. 161 (1996) 235-242.

9. H.B.Walikar, B.D.Acharya and E.Sampathkumar, Recent advances in the theory of domination in graphs and its applications, MRI Lecture Notes in Mathematics, Allahabad, No. 1, 1979. 